\title{
Controle das temperaturas de armazenamento e de distribuição de alimentos em restaurantes comerciais de uma instituição pública de ensino
}

\section{Control of temperature storage and distribution of food in commercial restaurants of a public education institution of}

Marlene Azevedo Magalhães Monteiro'

Rita de Cássia Ribeiro'

Bárbara Dora Alves Fernandes'

Júlia Ferreira de Rosa Sousa'

Lucília Moreira Santos'

' Curso de Nutrição, Universidade Federal de Minas Gerais. Belo Horizonte, MG, Brasil.

Correspondência / Correspondence Marlene Azevedo Magalhães Monteiro E-mail:marleneaz@enf.ufmg.br

\section{Resumo}

O objetivo do estudo foi avaliar as temperaturas de armazenamento e de distribuição de alimentos em restaurantes comerciais localizados numa instituição pública de ensino localizada município de Belo Horizonte-MG, e verificar sua conformidade em relação à legislação vigente. Utilizou-se um termômetro digital infravermelho; a coleta de dados foi realizada em seis restaurantes para a etapa de armazenamento (resfriados e congelados) e em 11 para a etapa de distribuição de preparações quentes e frias. Nesta, as aferições das temperaturas foram realizadas imediatamente após o término do seu preparo e a cada 30 minutos por um período total de 90 minutos (quatro aferições/dia), em três dias não consecutivos. Dos seis restaurantes avaliados para armazenamento de alimentos congelados e resfriados, quatro e cinco apresentaram temperatura adequada, respectivamente. Em relação à distribuição, apenas um apresentou temperatura adequada para as preparações quentes; em todos se encontrou inadequação para as preparações frias. Concluiu-se que o monitoramento da temperatura durante a distribuição dos alimentos é fator essencial para a qualidade das refeições e é urgente a implantação de mecanismos de controle, a fim de minimizar os riscos de contaminação.

Palavras-chave: Controle de Qualidade. Temperatura. Unidades Produtoras de Refeições. Manipulação de Alimentos. 


\section{Abstract}

This study aimed to evaluate the storage temperature and distribution of food in commercial restaurants located in a public educational institution located in the city of Belo Horizonte, State of Minas Gerais, Brazil, and verify its conformity to the legislation. A digital infrared thermometer was used; data collection was performed in six restaurants to step storage (chilled and frozen) and 11 for the step distribution of hot and cold preparations. In this, measurements of temperatures were taken immediately after completion of their preparation and every 30 minutes, for a total period of 90 minutes (four measurements / day) in three non-consecutive days. Among six restaurants for storage of frozen and chilled foods, four and five had adequate temperature, respectively. Regarding distribution, only one had adequate preparations for the hot temperature; all found inadequate for cold preparations. It was concluded that monitoring of temperature during distribution of food is essential to the quality of the meals and it is urgent to implement control mechanisms in order to minimize the risk of contamination.

Key words: Quality Control. Temperature. Restaurant. Food Handling.

\section{Introdução}

O setor de alimentação coletiva está em constante expansão no Brasil e sua importância econômica pode ser expressa na geração de empregos diretos; em número de refeições produzidas; na movimentação financeira mediante comercialização das refeições e no consumo de alimentos, o que representa para o governo uma receita de um bilhão de reais entre impostos e contribuições. Em 2012 o mercado de refeições coletivas no Brasil teve um faturamento de $\mathrm{R} \$ 14,7$ bilhões, alta de 13\% sobre o ano anterior, resultado de 11 milhões de refeições servidas pelas empresas do segmento. ${ }^{1}$

Dentre os vários aspectos relativos à crescente demanda pelos serviços de refeição fora do lar, a qualidade sanitária dos produtos oferecidos configura, ainda, uma questão fundamental, principalmente considerando a amplitude do público atendido. ${ }^{2,3}$ Nesse sentido, diversos procedimentos devem ser adotados para a garantia de um produto final adequado e livre de agentes patógenos, como obtenção de matérias-primas não contaminadas, práticas adequadas de manipulação e de higiene durante a preparação, equipamentos e estruturas operacionais eficientes e capacitação dos manipuladores de alimentos. ${ }^{3-8}$ 
Nesse contexto, destaca-se a importância da manutenção de temperaturas adequadas nas diferentes etapas de produção, o que implica diretamente a qualidade microbiológica das refeições. ${ }^{7,8}$ Assim, para que seja garantida a qualidade das refeições, é indispensável ficar atento ao binômio "tempo e temperatura”, fator muito importante na distribuição de refeições. Em vários restaurantes, as preparações ficam expostas no balcão térmico por longo período e, na maioria das vezes, sob temperatura inadequada, o que influencia de forma decisiva no crescimento da atividade microbiana. ${ }^{9}$

O objetivo deste trabalho foi avaliar as temperaturas de armazenamento e distribuição de alimentos em restaurantes comerciais localizados em uma instituição pública de ensino, e sua conformidade com a legislação vigente.

\section{Metodologia}

Trata-se de estudo descritivo e observacional realizado em restaurantes comerciais localizados no campus de uma instituição pública de ensino do município de Belo Horizonte-MG. Todos os 21 restaurantes/lanchonetes localizados no campus foram convidados a participar do estudo. No entanto, apenas seis aceitaram participar da etapa de avaliação das temperaturas de estocagem, e 11 da distribuição. Todos os responsáveis pelos estabelecimentos assinaram o Termo de Consentimento Livre e Esclarecido.

As aferições das temperaturas de estocagem ${ }^{8,10,11}$ dos produtos resfriados (leite e derivados, embutidos, sanduíches, molhos, frutas, legumes, folhosos e carnes) e congelados (carnes, peixes, alimentos industrializados, legumes, embutidos, massas e salgados) foram realizadas em três dias não consecutivos em seis restaurantes, conforme autorização prévia do responsável pelo local. Essa avaliação foi realizada em triplicata, com o objetivo de avaliar todos os fornecedores de alimentos, bem como maior diversidade de produtos entregues no restaurante.

Para as preparações frias (folhosos, verduras e legumes) e quentes (arroz, feijão, carnes, farofas, legumes e massas), as aferições das temperaturas ${ }^{8,10,11}$ foram realizadas imediatamente após o término do seu preparo e a cada 30 minutos por um período total de 90 minutos (quatro aferições/ dia), em três dias não consecutivos, em 11 restaurantes. Além disso, analisaram-se o tipo e a quantidade de equipamentos disponíveis e utilizados na manutenção da temperatura dos alimentos.

Para a coleta dos dados, utilizou-se um termômetro digital infravermelho com faixa de temperatura $-49^{\circ} \mathrm{C}$ a $230^{\circ} \mathrm{C}$, a uma distância de $20 \mathrm{~cm}$ do ponto central dos alimentos. ${ }^{5,710-12}$ Por fim, calculou-se a média para cada restaurante, média e desvio-padrão entre todos os estabelecimentos pesquisados, e valor mínimo e máximo das temperaturas, sendo os resultados foram confrontados com a legislação vigente. 


\section{Resultados e discussão}

$\mathrm{Na}$ análise das temperaturas dos alimentos resfriados e congelados, verificou-se adequação em 83,3\% ( $\mathrm{n}=5)$ dos restaurantes avaliados, de acordo com a Manual ABERC, ${ }^{10}$ com temperaturas inferiores a $6^{\circ} \mathrm{C}$ para carnes e $10^{\circ} \mathrm{C}$ para os demais produtos resfriados e $-18^{\circ} \mathrm{C}$ para os congelados.

Em relação à temperatura de distribuição das preparações frias, observaram-se inadequações em 100,0\% ( $\mathrm{n}=11)$ dos restaurantes avaliados, ou seja, acima de $10^{\circ} \mathrm{C}$, de acordo com a RDC $\mathrm{n}^{\mathrm{o}}$ $216 / 2004^{11}$ (tabela 1 ). A média geral entre os restaurantes foi de $20,8^{\circ} \mathrm{C}\left( \pm 3,0^{\circ} \mathrm{C}\right)$, e os valores mínimo e máximo foram, respectivamente, $17,0^{\circ} \mathrm{C}$ e $24,5^{\circ} \mathrm{C}$, o que expressa grande variação entre os restaurantes.

Ressalta-se que esses resultados podem ter ocorrido devido à presença de alimentos cozidos em saladas, preparados em horários próximos ao da distribuição, sem a submissão dos mesmos a processo de refrigeração adequado. E ainda, a ausência de um controle efetivo da temperatura dos balcões refrigerados possivelmente contribuiu para esses resultados.

De acordo com a Portaria CVC 05/2013, ${ }^{12}$ estas preparações poderiam alcançar temperaturas entre $10^{\circ} \mathrm{C}$ e $21^{\circ} \mathrm{C}$ por um período máximo de duas horas. Por esta análise, seis restaurantes avaliados estariam adequados.

Outros autores analisaram temperatura de pratos frios servidos em restaurantes comerciais. Alves \& Mesquita, ${ }^{13}$ em estudo realizado em Santa Maria-RS, verificaram temperaturas inadequadas, principalmente para os folhosos. Tal fato foi associado ao excessivo tempo de manipulação e a proximidade de fornos e fogões, que desencadearam aumento da temperatura. Em outro estudo, Marinho et al. ${ }^{9}$ também verificaram a ausência de adequação das temperaturas de preparações frias em uma Unidade de Alimentação e Nutrição (UAN) de grande porte localizada em Belo Horizonte-MG, associada ao excessivo tempo de manipulação e ao baixo tempo de refrigeração anterior à distribuição, como observado neste estudo. Carvalho et al. ${ }^{14}$ avaliaram três restaurantes comerciais de Goiânia-GO, e da mesma forma obtiveram resultados insatisfatórios para as preparações frias prontas.

Para as preparações quentes, observou-se que apenas em um restaurante $(9,0 \%)$ foram encontrados valores adequados de temperatura, sendo igual ou superior a $65^{\circ} \mathrm{C}$, de acordo com a RDC no 216/2004 ${ }^{11}$ (tabela 1). Outros três estabelecimentos (27,0\%) obtiveram temperatura adequada somente em um dos dias aferidos, e os demais $(n=7,64 \%)$ apresentaram temperaturas inadequadas todos os dias.

A média geral entre os restaurantes foi de $56,0( \pm 7,1)$, e os valores mínimo e máximo foram $42,4^{\circ} \mathrm{C}$ e $69,4^{\circ} \mathrm{C}$, respectivamente, o que demonstra grande variação entre os estabelecimentos 
pesquisados. Provavelmente, os fatores que contribuíram para essa variação entre os restaurantes foram a ausência de controle da temperatura da espera para a distribuição, equipamentos inadequados de aquecimento e a falta de mecanismos de controle.

De acordo com a Portaria CVS05/2013, ${ }^{12}$ os alimentos quentes podem ficar na distribuição ou espera a $60^{\circ} \mathrm{C}$ ou mais por no máximo seis horas, e abaixo de $60^{\circ} \mathrm{C}$ por no máximo uma hora. Ao adotar esses parâmetros, apenas três restaurantes encontravam-se adequados.

Em alguns restaurantes, o desvio-padrão foi alto. Este resultado pode ser devido às especificidades das preparações do cardápio do dia da análise, bem como deficiências nos equipamentos para manutenção da temperatura e o balcão térmico.

Tabela 1. Valores médios e desvio-padrão das temperaturas $\left({ }^{\circ} \mathrm{C}\right)$ das preparações frias e quentes após seu preparo nos restaurantes comerciais localizados no campus de uma instituição pública de ensino. Belo Horizonte-MG, 2012.

\begin{tabular}{ccc}
\hline \multirow{2}{*}{ Restaurantes } & \multicolumn{2}{c}{ Preparações } \\
\cline { 2 - 3 } 1 & Frias & Quentes \\
\hline 2 & $19,7( \pm 1,3)$ & $49,5( \pm 0,8)$ \\
3 & $24,5( \pm 0,4)$ & $52,4( \pm 6,2)$ \\
4 & $24,4( \pm 1,2)$ & $42,4( \pm 3,4)$ \\
5 & $18,0( \pm 0,5)$ & $61,6( \pm 1,5)$ \\
6 & $24,0( \pm 0,2)$ & $53,6( \pm 4,4)$ \\
7 & $20,7( \pm 0,5)$ & $69,4( \pm 1,7)$ \\
8 & $21,6( \pm 0,6)$ & $59,8( \pm 7,8)$ \\
9 & $17,5( \pm 0,5)$ & $55,7( \pm 11,9)$ \\
10 & $23,5( \pm 1,3)$ & $52,9( \pm 4,4)$ \\
11 & $17,6( \pm 4,5)$ & $57,2( \pm 3,7)$ \\
& $17,0( \pm 1,8)$ & $61,0( \pm 14,9)$ \\
\hline
\end{tabular}


Resultados semelhantes a este estudo foram obtidos por Frantz et al., ${ }^{15}$ ao avaliarem registros de processos em UANs. Eles constataram, de um modo geral, que as preparações quentes obtiveram maior adequação de temperatura em relação às preparações frias.

Barbieri et al., ${ }^{16}$ em estudo realizado numa UAN localizada na zona sul da cidade do Rio de JaneiroRJ, concluíram que a temperatura de exposição da guarnição e das carnes foi inferior algumas vezes à temperatura ideal, devido a fatores como o preparo dos alimentos para reposição ser realizadocom antecedência e não armazenado de forma adequada.

Chesca et al., ${ }^{17}$ quando avaliaram as temperaturas de distribuição de refeições frias e quentes em restaurantes de Uberaba-MG, observaram que 25,0\% das preparações quentes estavam abaixo de $60^{\circ} \mathrm{C}$.

Em outro estudo, de Soares, Monteiro \& Schaefer, ${ }^{18}$ o controle do tempo de distribuição de preparações quentes em um restaurante universitário indicou que todas as preparações atendiam ao estabelecido pela legislação brasileira, pois mesmo aquelas com temperaturas inadequadas não permaneciam expostas ao consumo por um período superior ao recomendado pela CVS-6/1999. ${ }^{12}$

Por fim, de uma forma geral, a presença de equipamentos como pass through, banho-maria ou geladeiras industriais, e programas de manutenção preventiva, contribuiriam para reduzir e/ou manter a temperatura das preparações frias e quentes no balcão de distribuição, além de processos de controle de temperatura.

Uma das limitações deste estudo foi a grande resistência dos funcionários dos restaurantes em permitir a coleta de dados, sob a alegação de interferência na rotina das atividades do restaurante.

\section{Conclusão}

A temperatura adequada, tanto no armazenamento como na distribuição, é um dos fatores que podem contribuir para a garantia da qualidade das refeições servidas. Por isso, deve ser monitorada constantemente, minimizando os riscos de contaminação e crescimento microbiológico e melhorando a qualidade das preparações servidas nos restaurantes.

Devem ser estabelecidos procedimentos de controle diário e medidas corretivas ou preventivas, de forma a promover o monitoramento adequado das temperaturas.

\section{Agradecimentos}

À Seção de Fiscalização de Contratos e Serviços da Divisão de Serviços Comunitários do Departamento de Serviços Gerais da Universidade Federal de Minas Gerais. 


\section{Referências}

1. Associação Brasileira de Refeições Coletivas - ABERC. História e mercado. São Paulo: ABERC; 2013. [acesso 04 jun. 2013]. Disponível em:<htpp://www.aberc.com.br>

2. Salay E. Consumo alimentar fora do domicílio: implicações para pesquisas em segurança alimentar e nutricional. Com Ciência [Internet]. 2005. [acesso em 05 nov. 2009]. Disponível em: http://www. comciencia.br/reportagens/2005/09/14.shtml

3. Baltazar C, Shimozako HJ, Amaku M, Pinheiro SR, Perondi AMT. Avaliação higiênico-sanitária de estabelecimentos da rede Fast Food no Município de São Paulo. Hig Aliment. 2006; 20(142):46-51.

4. Matos $\mathrm{CH}$ de, Proença RPC. Condições de trabalho e estado nutricional de operadores do setor de alimentação coletiva: um estudo de caso. Rev Nutr. 2003; 16(4):493-502.

5. Nascimento Neto F do. Roteiro para elaboração de manual de boas práticas de fabricação (BPF) em restaurantes. 2ed. São Paulo: Editora Senac-SP; 2005.

6. Proença RPC, Sousa AA, Veiros MB, Hering B. Qualidade nutricional e sensorial na produção de refeições. Florianópolis: Editora da UFSC; 2005.

7. Chaves JBP, Assis FCC, Pinto NBM, Seixas PS. Boas práticas de fabricação (BPF) para restaurantes, lanchonetes e outros serviços de alimentação. Viçosa, MG: Ed. UFV; 2006.

8. Silva Junior EA. Manual de controle higiênico: sanitário em serviços de alimentos. $\sigma^{a}$ ed. São Paulo: Varela; 2002.

9. Marinho CB, Souza CS, Ramos SA. Avaliação do binômio tempo-temperatura de refeições transportadas. e-scientia. 2009; 2(1):1-11.

10. Associação Brasileira de Refeições Coletivas. Manual ABERC de práticas de elaboração e serviço de refeições para coletividades. 9a ed. São Paulo: ABERC; 2009.

11. Brasil. Agência Nacional de Vigilância Sanitária. Resolução RDC n. 216, 15 set. 2004. Dispõe sobre Regulamento Técnico de Boas Práticas para Serviços de Alimentação. Diário Oficial da União. 16 set. 2004.

12. São Paulo. Centro de Vigilância Sanitária. Secretaria de Estado da Saúde. Portaria CVS-5/13, de 09/04/2013. Regulamento Técnico de Boas Práticas para Estabelecimentos Comerciais de Alimentos e para Serviços de Alimentação, e o Roteiro de Inspeção. [acesso 30 jan 2013]. Disponível em: < http:// www.cvs.saude.sp.gov.br/up/PORTARIA\%20CVS-5_090413.pdf>

13. Alves M, Mesquita MO. Monitoramento da temperatura de preparações frias de uma Unidade de Alimentação e Nutrição Comercial da Cidade de Santa Maria-RS. In: Promovendo Saúde na Contemporaneidade: desafios de pesquisa, ensino e extensão; 08-11 jun. 2010; Santa Maria, Brasil.

14. Ricardo FO, Moraes MP, Carvalho ACMS. Controle de tempo e temperatura na produção de refeições de restaurantes comerciais na cidade de Goiânia-GO. Demetra. 2012; 7(2):85-96.

15. Frantz CB, Bender B, Oliveira ABA, Tondo EC. Avaliação de registros de processos de quinze unidades de alimentação e nutrição. Alim. Nutr. 2008; 19(2):167-175. 
16. Barbieri RR, Esteves AC, Matoso R. Monitoramento da temperatura de preparações quentes e frias em uma unidade de alimentação e nutrição. Hig. Alim. 2011; 25(194-195):40-45.

17. Chesca AC, Caetano AM, Leite APC, Polveiro AM, Terra AD, Lyra FS, Zaidan MCC, Okura MH. Avaliação das temperaturas de pistas frias e quentes em restaurantes da cidade de Uberaba, MG. Hig Aliment. 2001; 15(87):38-43.

18. Soares ADN, Monteiro MAM, Schaefer MA. Avaliação do binômio tempo e temperatura em preparações quentes de um Restaurante Universitário. Hig Aliment. 2009; 23(174/175):36-41.

Recebido: 15/7/2013

Revisado: 02/11/2013

Aprovado: 06/1/2014 